\title{
Leakage current in atomic-size surface interconnects
}

\author{
Mikaël Kepenekian, ${ }^{1,2, a)}$ Roberto Robles, ${ }^{1,2}$ Christian Joachim, ${ }^{3,4}$ and Nicolás Lorente ${ }^{1,2}$ \\ ${ }^{1}$ ICN2-Institut Catala de Nanociencia i Nanotecnologia, Campus UAB, 08193 Bellaterra (Barcelona) Spain \\ ${ }^{2}$ CSIC-Consejo Superior de Investigaciones Cientificas, ICN2 Building, Campus UAB, 08193 Bellaterra \\ (Barcelona) Spain \\ ${ }^{3}$ GNS and MANA satellite, Centre d'Elaboration des Matériaux et d'Etudes Structurales (CEMES), CNRS, \\ 29 rue J. Marvig, 31055 Toulouse Cedex, France \\ ${ }^{4}$ Institute of Materials Research and Engineering, 3 Research Link, Singapore 117602, Singapore
}

(Received 19 July 2013; accepted 3 October 2013; published online 16 October 2013)

\begin{abstract}
The current lost into a doped silicon substrate from a surface-supported nanowire is evaluated using transport calculations based on density functional theory. The calculations are performed for an infinite non-periodic wire for various types of dopants. Two concentration limits are explored: the single-dopant and the massively doped limits. Our calculations permit us to conclude that n-doped $\mathrm{Si}$ will be less leaky than p-doped Si. For the low bias at which these nanodevices will operate, leakage currents will be less than $10 \%$ for n-doped Si substrates and $20 \%$ for p-doped ones.

(C) 2013 AIP Publishing LLC. [http://dx.doi.org/10.1063/1.4825375]
\end{abstract}

Silicon-based atomic-size circuitry is rapidly progressing. In the last decade, proofs-of-concept and devices have been created. Single-atom quantum dots, based on surface silicon dangling bonds (DB), have been shown to be a promising technological venue. ${ }^{1}$ Recently, structures formed by DB quantum dots have been shown to have qubit capabilities. $^{2}$ Arrays of aligned DB have been created and tailored to present specific electronic properties, ${ }^{3}$ and single-atoms have been precisely positioned in atomically thin epitaxial circuits to create an atomic transistor. ${ }^{4}$ Among this ongoing revolution in the creation of a silicon-based atomic-scale technology, interconnects are playing an important role: Electronic currents can be naturally driven in and out the atomic-scale devices via atomic-size nanowires. Freestanding ${ }^{5}$ or supported ${ }^{6}$ silicon nanowires have proved to be very good conductors. Other types of nanowires, such as arrays of aligned DB, are also promising candidates. ${ }^{7}$ The pace of this evolution is fast enough to raise issues that will become pressing in order to have a working technology.

One such issue is the problem of current leakage. In traditional transistor-based circuitry, the leakage of current through the gate dielectric is a limiting factor and object of continuing research. ${ }^{8}$ However, the paradigm of atomic-size circuitry leads to another type of current leakage. Indeed, atomic devices are necessarily coupled to their environment. This coupling perturbs the device in different ways. The dielectric mismatch between nanowire and surroundings has been shown to reduce in 50\% the held current. ${ }^{9}$ More limiting can be the leakage of the electronic current driving the device. This leakage can seriously disrupt the original properties of the devices and bring to a halt the recent extraordinary technological progress. It is then important to assess the features and parameters controlling current leakage in atomic-size circuitry.

In this letter, we present a study of current leakage on supported DB atomic scale wires (hereinafter referred to as DB wires), performed using density-functional-theory (DFT)

${ }^{\text {a)} E l e c t r o n i c ~ m a i l: ~ m i k a e l . k e p e n e k i a n @ c i n 2 . e s ~}$ electronic transport calculations. These nanowires are either epitaxially fabricated ${ }^{10}$ or crafted $^{3,11-13}$ on H-passivated silicon surfaces. Dopants determine the final transport properties of DB's by fixing the Fermi energy. ${ }^{14,15}$ But they also permit charge transport in bulk silicon. Hence, DB atomic scale wires supported by a doped substrate will not be electronically confined and their transport capabilities can be seriously compromised.

We use the TRANSIESTA package ${ }^{16}$ to compute electron currents of silicon DB wires. ${ }^{17}$ Briefly, a surface $2 \times 1$ unit cell was used to accommodate one single $\mathrm{Si}(100)$ dimer. The slab supercell structure contained 8 bi-layers in the direction perpendicular to the slab. The atomic structure was relaxed until forces were smaller than $0.04 \mathrm{eV} / \AA$ using an atomic double- $\zeta$ with polarization basis set. The transport calculations used a single- $\zeta$ with polarization basis set. There is no periodicity along the transport direction and three different regions of the circuit are considered: (i) semi-infinite left electrode, (ii) contact region, and (iii) semi-infinite right electrode, following the scheme of Figure 1. The conductance calculations have been done using the Landauer formula with non-equilibrium Green's functions. ${ }^{16}$ The main input of the present work is the atomic construction of each region because depending on whether dopants are included or not the transport properties will be very different. A DB wire is built by removing one of the two hydrogens of the single dimer of the unit cell. Each of the three regions contains 4 dimers in the transport direction and 2 in the other direction where the periodicity is conserved, assuring a decoupling between neighboring DB nanowires. ${ }^{17}$ Furthermore, we perform a spinless calculation; despite not being the ground state, ${ }^{11,18-21}$ the electronic structure of the studied DB wire corresponds to the perfectly metallic one that has been previously studied. ${ }^{17,22,23}$

Dopants are included by replacing one $\mathrm{Si}$ atom in the relevant transport region, Figure 1, by either a boron (p-doping) or a phosphorous (n-doping) atom. This substitution can be achieved at different distances from the surfaces, allowing one to test the extent of the effect of the dopant. The new 
atomic coordinates correspond to a complete ionic relaxation of the passivated surface in order to avoid further reconstruction of the DB nanowire. With the setup of Figure 1, we can have three different combinations: (a) where only the contact region (ii) is doped, or (b) with dopants in the three regions, or (c) with dopants in two regions, one being the contact region (ii). These combinations allow us to calculate different currents leading to an evaluation of the current leakage. In order to achieve this, we observe that the system of Figure 1(c) consists of an electrode without doping such that, at low bias, the only open channels are the ones of the nanowire. Hence, the current must necessarily proceed via the DB wire since there is no dopant current contribution. This setup acts like a filter that selects the electrons that flow through the DB wire as the only ones forming the current. We call this contribution $I_{\text {filter }}$. Meanwhile Figure 1(b) has no restriction and would correspond to a total current $I_{\text {total }}$ that is composed of a dopant current contribution $I_{\text {dopant }}$ and the DB wire contribution $I_{\text {filter }}^{\prime}$ which is different from the previous $I_{\text {filter }}$. Figure 1 setup (a) is again nanowire confined except that the transport channels are perturbed by the presence of dopants in the contact region leading to the contribution $I_{\text {impurity. }}$.

Setups (a) and (b) can be thought of as the same setup in two different doping limits. In case (b), we can think that there is another undoped electrode asymptotically far to the left, hence we are just effectively increasing the size of the contact region of (a). Our transport calculations in these setups permit us to compute the effect of dopants in two opposing limits: the single impurity or dopant limit for case (a) and the massively doped case (b) where one Si atom out of nearly 600 is replaced by a dopant (B or $\mathrm{P}$ ). In the absence of dopants, the current will just be the full nanowire's current that we take as the current reference, $I_{\text {ref }}$, since it is the maximum current the nanowire can convey. Then the leakage current will be $I_{\text {leakage }}=I_{\text {ref }}-I_{\text {impurity }}$ for (a) and $I_{\text {leakage }}=I_{\text {ref }}-I_{\text {filter }}$ for (b) and (c).

The currents are evaluated following Landauer's equation: ${ }^{24}$

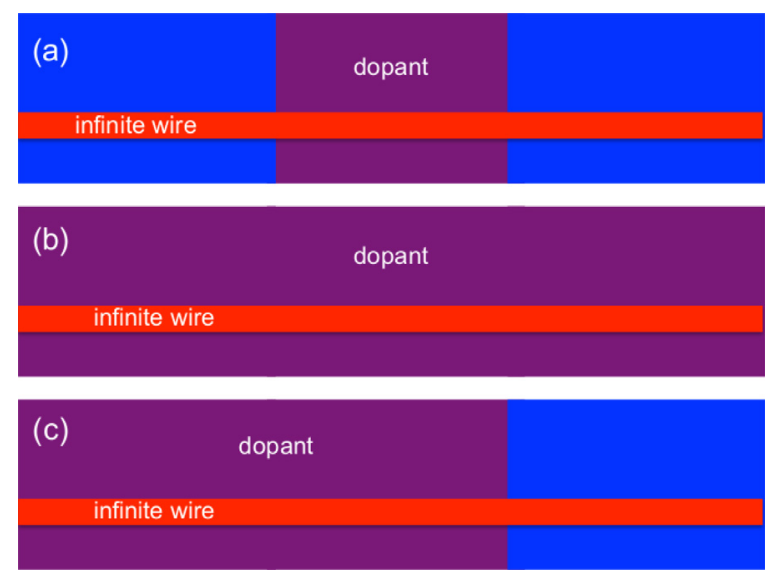

FIG. 1. Top schematic view of the transport calculation setups. The atomic structures are arranged in two semi-infinite electrodes and a central connecting region between the electrodes. In (a) only the central region is doped, in (b) there are dopants in the three regions, and in (c) dopants are found in two regions, one being the contact region. The doping takes place in the substrate and the infinite, non-periodic nanowires extend from the left to the right electrodes. Considered dopants are B and P atoms substituting a Si bulk atom.

$$
I=\frac{2 e}{h} \int_{-\infty}^{\infty} T(E, V)\left[f_{R}(E)-f_{L}(E)\right] d E,
$$

where $T(E, V)$ is the transmission function for an electron of energy $E$ when the bias between the two DB electrodes is $V$, and $f_{R}(E)\left(f_{L}(E)\right)$ is the right- (left-) electrode Fermi occupation function. We further simplify the current $I$ calculation using the zero-bias transmissions.

Figure 2 shows the transmissions, $T(E, V=0)$, for the single dopant (Figure 2(a) B-doped and 2(b) P-doped). The distance of the dopant to the DB wire affects the degree of perturbation on the transmission, but within the used distances $(15,9.5$, and $4 \AA$ ) the qualitative behavior is the same. For B impurities, the negative-energy transmission is very affected due to the interaction of the DB wire's electronic structure with the energy levels of the B ion itself. However, the positive region transmission is also disrupted, and indeed basically one transmission channel disappears above $0 \mathrm{eV}$. This situation is quite different from $\mathrm{H}$ impurities along the DB wire. ${ }^{17}$ A transmission eigenchannel analysis ${ }^{25}$ shows that while $\mathrm{H}$ was perturbing the two available channels of the $\mathrm{DB}$ wire, $\mathrm{B}$ is basically quenching one of the channels. $\mathrm{P}$ ions have higher-energy levels and consequently the main effect takes place at positive energies. From Figure 2 it is clear that $\mathrm{P}$ ions perturb the transmission less than $\mathrm{B}$ ions and we should expect a lower leakage current for devices crafted onto n-doped samples.

Figures 2(c) and 2(d) show the transmissions, $T(E, V=0)$, for the massively doped limit ((c) $\mathrm{B}$ and (d) $\mathrm{P}$ dopants). Blue curves correspond to setup (b) and red to (c),

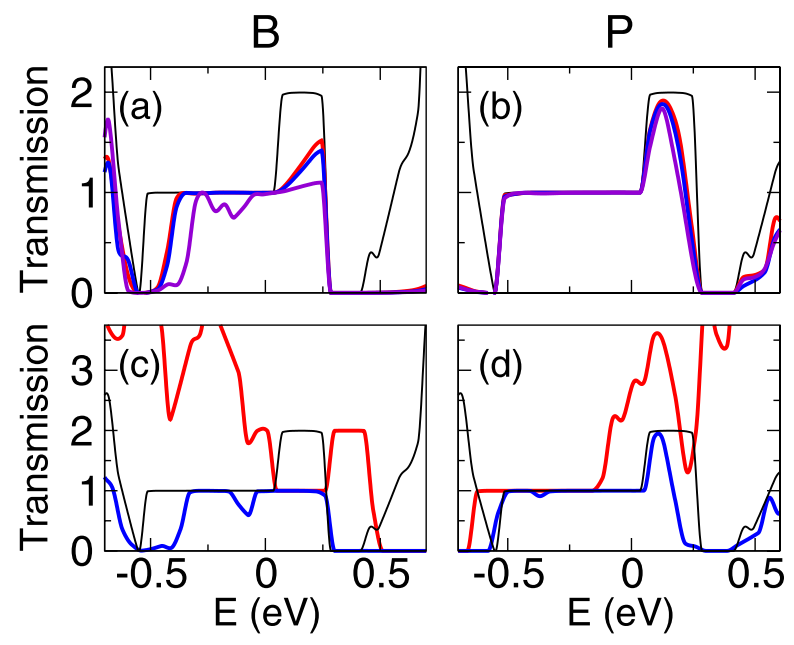

FIG. 2. Electron transmissions from the left to the right electrode as a function of electron energy referred to the Fermi energy. In all panels, the nanowire's transmission in the absence of dopants is represented by the black full-line curve. (a) Transmission with a single substitutional B-atom as dopant for located at $15 \AA$ (red curve), $9.5 \AA$ (blue curve), $4 \AA$ (mauve curve) underneath the nanowire. (b) Single substitutional P-atom dopant for the same distances and color code as (a). (c) Massively doped system with $\mathrm{B}$-atoms in the left electrode plus contact region (blue line) and also in the right electrode (red line), the dopants are located at $4 \AA$ underneath the nanowire. (d) Same as (c) but using P-atoms. Transmissions for the same type of dopant are largely independent of the dopant concentration as soon as one of the electrodes is not doped (see the mauve curve of (a) and the blue line in (c), the same for (b) and (d)). Red lines in (c) and (d) are dominated by the dopant current contribution, and they strongly depend on the amounts of dopants. There are, however, a few regions where transport is purely nanowire confined. 

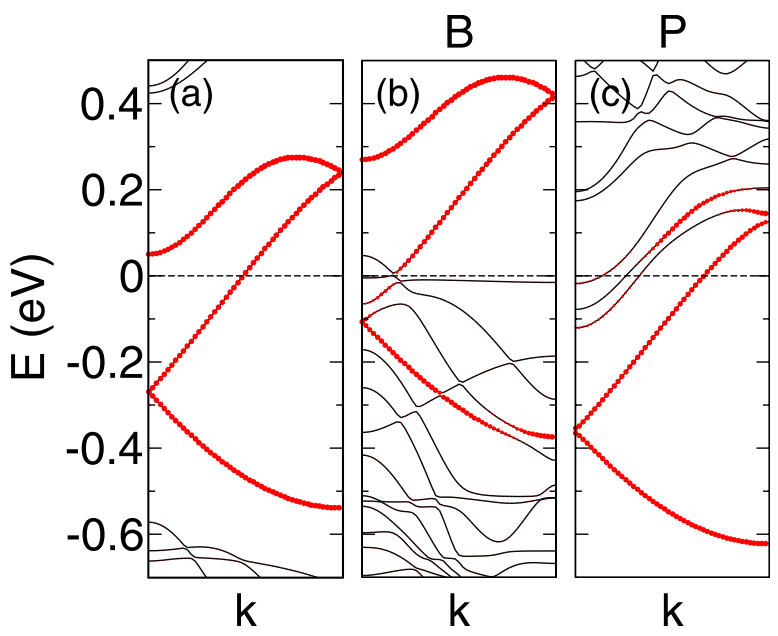

FIG. 3. Electronic bands evaluated for a periodic nanowire with (a) a pure Si substrate, (b) B-doped Si substrate, and (c) P-doped Si substrate. The red circles indicate the weight of the DB in the bands. The Fermi energy is taken as a reference. Doping shifts the Fermi energy but more importantly it brings about electronic mixing between nanowires states and bulk states as shown in the avoided crossings of (b) and (c).

they are then the nanowire transmission and nanowire plus dopant transmission, respectively. Remarkably, the qualitative features of the single impurity case are retrieved for the nanowire transmission, due to the very confined (atom-wide) transport through the DB wire. On the other hand, the electron transmission for the fully doped case, setup (c), is dominated by the dopant contribution. Slightly transforming the setup can lead to a periodic system in which the electronic band structure along the transport direction can be evaluated. This is shown in Figure 3. The left panel shows the undoped case, the center panel the B-doped one, and the right panel the corresponding P-doped case. The mixture between the nanowire band (left panel) and the bulk bands become obvious, mainly taking place at negative energies for B and at positive for $\mathrm{P}$ as discussed above for the single impurity. This study permits us to conclude that the cause of the current leakage is the disruption of the DB wire as an electronic entity separated from the bulk. Indeed, the dopants mix up DB wire's and bulk states causing the electron leak from the wire.

The relative amounts of leakage current can be evaluated from the current expression, Eq. (1). Figure 4 shows the leakage current normalized to the current of the undoped wire. Astonishingly, we find that the percentage of leakage currents is largely independent of the dopant concentration. In order to assess the importance of the dopant-wire distance we have computed different leakage currents for different distances. This is the difference between the graphs plotted on the up and down lines of Figure 4. We see that as the dopant concentration near the wire decreases, the leak diminishes but again, single or many dopants yield comparable results. These results show that a single dopant located within tens of Ångströms of a DB wire is enough to cause a substantial mixing of the DB wire's electronic structure with the substrate's one, leading to most of the expected leakage in these systems. In agreement with the transmission functions and due to the particular electronic structure of the doping ions, we retrieve that $\mathrm{B}$ has a more sustained effect on

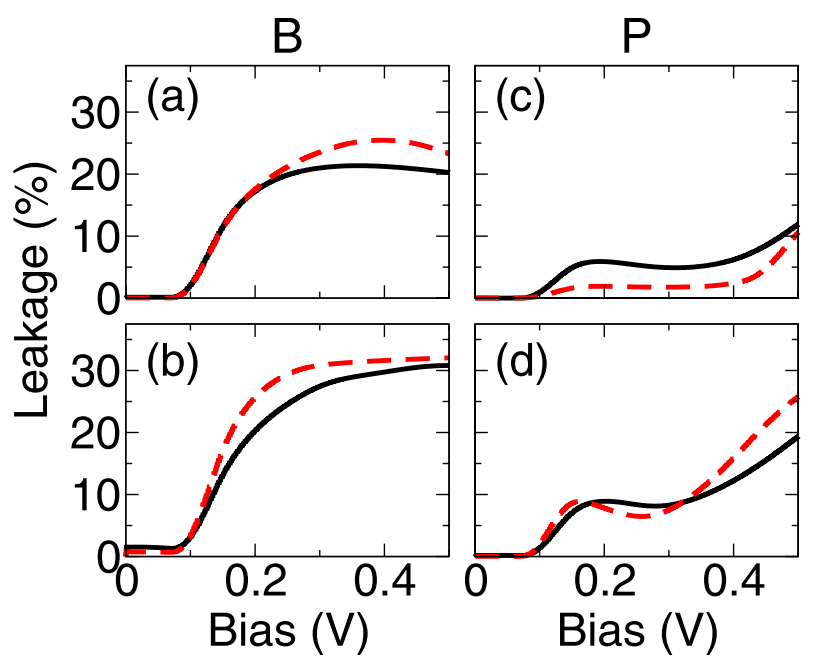

FIG. 4. Ratio between the current lost into the Si bulk (leakage current) and the nanowire's current in the absence of dopants. (a) For a B-atom replacing a $\mathrm{Si}$ atom $15 \AA$ away deep in the Si bulk (full line: single dopant limit, dashed line: massively doped limit). (b) For a B-atom $4 \AA$ underneath the nanowire. (c) For a P-atom $15 \AA$ away deep in the Si bulk. And (d) for a $\mathrm{P}$-atom $4 \AA$ underneath the nanowire. The higher energies of $\mathrm{P}$ electronic levels perturb less the nanowire's states. For low-bias, P-atom doping leads to leakage currents below $10 \%$ the wire's electronic transport capabilities.

the current leakage than P. Indeed, even in the worse case scenario, our simulations permit us to conclude that P-doped substrates can hold interconnects with a current leakage reduced to less than $20 \%$. For low bias, this figure improves to less than $10 \%$. B-doped substrates present a slightly larger current leakage of $\sim 30 \%$. Due to the low-lying electronic levels of B ions, the current leakage is already sizable at low bias for B-doped substrates.

In summary, we have performed simulations of the leakage of the electronic current of surface based interconnects in the presence of dopants. Our simulations consider two very different limits: the unique dopant and the massively doped substrates. Due to the local nature of the interactions we find very similar results in both limits, permitting us to gauge the extent of damage in the electronic conduction capabilities of surface interconnects. In the case of metallic dangling-bond wires, our conclusion is that for low bias, Pdoped substrates are preferable since electronic leaks diminish to less than $10 \%$ of the total current, while for B-doped substrates the current can leak up to $20 \%$ in the same conditions. Surface interconnects and other silicon supported nanodevices will certainly benefit from $\mathrm{n}$-doped substrates.

The authors acknowledge financial support from the European-Union Integrated Project AtMol (http:// www.atmol.eu).

${ }^{1}$ M. B. Haider, J. L. Pitters, G. A. DiLabio, L. Livadaru, J. Y. Mutus, and R. A. Wolkow, Phys. Rev. Lett. 102, 046805 (2009).

${ }^{2}$ L. Livadaru, P. Xue, Z. Shaterzadeh-Yazdi, G. A. DiLabio, J. Mutus, J. L. Pitters, B. C. Sanders, and R. A. Wolkow, New J. Phys. 12, 083018 (2010).

${ }^{3}$ A. J. Schofield, P. Studer, C. F. Hirjibehedin, N. J. Curson, G. Aeppli, and D. R. Bowler, Nat. Commun. 4, 1649 (2013).

${ }^{4}$ M. Fuechsle, S. Mahapatra, F. A. Zwanenburg, M. Friesen, M. A. Eriksson, and M. Y. Simmons, Nat. Nanotechnol. 5, 502 (2010).

${ }^{5}$ Y. Cui and C. M. Lieber, Science 291, 851 (2001). 
${ }^{6}$ B. Weber, S. Mahapatra, H. Ryu, S. Lee, A. Fuhrer, T. C. G. Reusch, D. L. Thompson, W. C. T. Tee, G. Klimeck, L. C. L. Hollenberg, and M. Y. Simmons, Science 335, 64 (2012).

${ }^{7}$ M. Kepenekian, R. Robles, C. Joachim, and N. Lorente, Nano Lett. 13, 1192 (2013).

${ }^{8}$ B. S. Eller, J. Yang, and R. J. Nemanich, J. Vac. Sci. Technol. A 31, 050807 (2013).

${ }^{9}$ M. T. Björk, H. Schmid, J. Knoch, H. Riel, and W. Riess, Nat. Nanotechnol. 4, 103 (2009).

${ }^{10}$ A. Fuhrer, M. Fuechsle, T. C. G. Reusch, B. Weber, and M. Y. Simmons, Nano Lett. 9, 707 (2009).

${ }^{11}$ T. Hitosugi, S. Heike, T. Onogi, T. Hashizume, S. Watanabe, Z.-Q. Li, K. Ohno, Y. Kawazoe, T. Hasegawa, and K. Kitazawa, Phys. Rev. Lett. 82, 4034 (1999).

${ }^{12}$ T. Hallam, T. C. G. Reusch, L. Oberbeck, N. J. Curson, and M. Y. Simmons, J. Appl. Phys. 101, 034305 (2007).

${ }^{13}$ F. Bianco, J. H. G. Owen, S. A. Köster, D. Mazur, C. Renner, and D. R. Bowler, Phys. Rev. B 84, 035328 (2011).

${ }^{14}$ T. Reusch, O. Warschkow, M. Radny, P. Smith, N. Marks, N. Curson, D. McKenzie, and M. Simmons, Surf. Sci. 601, 4036 (2007).
${ }^{15}$ J. L. Pitters, P. G. Piva, and R. A. Wolkow, J. Vac. Sci. Technol. B 30, 021806 (2012).

${ }^{16}$ M. Brandbyge, J.-L. Mozos, P. Ordejón, J. Taylor, and K. Stokbro, Phys. Rev. B 65, 165401 (2002).

${ }^{17}$ M. Kepenekian, F. D. Novaes, R. Robles, S. Monturet, H. Kawai, C. Joachim, and N. Lorente, J. Phys.: Condens. Matter 25, 025503 (2013).

${ }^{18}$ C. F. Bird and D. R. Bowler, Surf. Sci. 531, L351 (2003).

${ }^{19}$ J.-H. Cho and L. Kleinman, Phys. Rev. B 66, 235405 (2002).

${ }^{20}$ J. Y. Lee, J.-H. Cho, and Z. Zhang, Phys. Rev. B 80, 155329 (2009).

${ }^{21}$ R. Robles, M. Kepenekian, S. Monturet, C. Joachim, and N. Lorente, J. Phys.: Condens. Matter 24, 445004 (2012).

${ }^{22}$ P. Doumergue, L. Pizzagalli, C. Joachim, A. Altibelli, and A. Baratoff, Phys. Rev. B 59, 15910 (1999).

${ }^{23}$ H. Kawai, Y. K. Yeo, M. Saeys, and C. Joachim, Phys. Rev. B 81, 195316 (2010).

${ }^{24}$ S. Datta, Electronic Transport in Mesoscopic Systems (Cambridge University Press, Cambridge, 1997).

${ }^{25}$ M. Paulsson and M. Brandbyge, Phys. Rev. B 76, 115117 (2007). 